\section{A Modified Microbiological Assay Procedure}

Ar a joint meeting of the Biochemical Society and the Society for General Microbiology, the principles underlying microbiological assay were discussed in some detail ${ }^{1}$. The advantages of measuring bacteriological growth directly, thus avoiding tho need to pass the lag period before making indirect measurements of growth, such as acidimetry, are offset by the greater complexity of apparatus and technique noeded in the more direct ones, such as turbidimetry.

During the discussion at the meeting, I suggested that it might be possible to make the best of both worlds by applying in reverse to the assay of vitamins and amino-acids the principles now very generally adopted for the assay of penicillin and other antibiotics. In other words, the medium would be devoid of the vitamin to be estimated and would be inoculated with the chosen strain of organism, graded amounts of the vitamin being then pipetted into suitably formed cups, using the ordinary Petri dish and agar medium as in the Heatley test and its various modifications. This should result in a circular area of growth round the cup, that is, in a 'zone of exhibition'.

It was not possible to investigate this matter for some time after the meeting mentioned; but Dr. W. F. J. Cuthbertson in these Laboratories has now been able to do so. We find that the zones are, indeed, graded to dose, being over a fairly wide range proportional in diameter to the logarithm of the amount of the test vitamin in the eup. The method seems to work not only for riboflavine and aneurine, on two different media, but also for several other vitamins of the $B$ group.

Among the several advantages of this method, which may be of wide application, there are two outstanding ones. In the first place, it puts at the disposal of the microbiological assayist a far wider range of organisms than hitherto, for he is no longer restricted to those that produce acid. This may mean, in effect, that he can bring into use for analytical purposes some of the less-exacting organisms that are known to require only one or two organic nutrients, apart from a source of carbohydrate with the usual mineral salts. The second outstanding advantage is speed, for it has been found not merely possible but desirable to use media and techniques giving maximum growth in less than 24 hours. The Petri dish procedure, furthermore, besides making replication easy, lends itself very well to tests of factorial design with subsequent analysis of variance in their statistical treatment. The chief disadvantage encountered is that, in comparison with the acidimetric technique in test tubes, considerably higher concentrations must be used, and the method as at present carried out cannot be applied to, for example, the estimation of riboflavine in flour and other wheat products.

Further work on the test is in hand, and it is hoped to publish a more detailed account at a later date.

\section{A. L. BACHARACH}

Glaxo Laboratories, Ltd., Greenford, Middlesex. Sept. 16.

${ }^{1}$ Biochem. J., 41, i (1947).

\section{Escape of Thoron from Thorium $X$ Salts}

THe absorption method devised by several author $3^{1,2,3}$ for obtaining the relative amounts of radium, mesothorium and radiothorium in the mixed salts of these elements in torms of their gamma-ray equivalent has been used for some sixteen sources of this kind destined for a radium mass-unit.

In fifteen of these sources the salts, forming a sphere $5 \mathrm{~mm}$. in diameter and enclosed in a steel tube with walls $1 \mathrm{~mm}$. thick (Fig. 1), emit gamma rays equivalent to $180 \mathrm{mgm}$. of radium, from which about 34 per cent belong effectively to the gamma rays of radium $\mathrm{B}+\mathrm{C}, 33$ per cent to mesothorium 2 and 33 per cent to thorium $\mathrm{C}+\mathrm{C}^{\prime \prime}$.

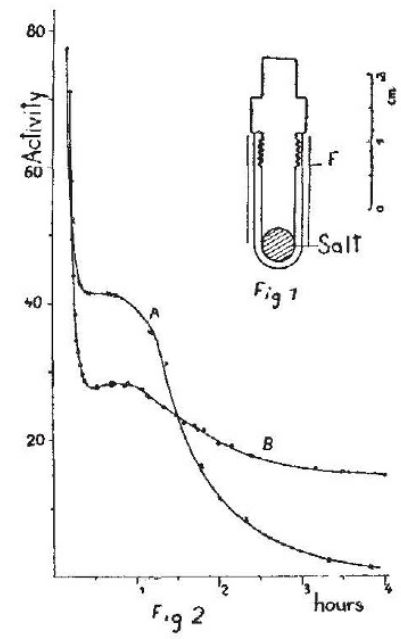

As several of these tubes leaked considerably after they had been sealed, an attempt was made to compare the amount of the escaping radon to that of the thoron by means of their active deposits. We hoped to obtain in this way some information concerning the relation between the parent elements of these two emanations.

In the first experiment the leaky container was exposed for 1 hour in an air-tight vessel (volume 1.5 1.) filled with air at normal pressure. After exposure, the air of the vessel was passed into an evacuated ionization chamber, measured with a bifilar electrometer, and after a total time of $5 \mathrm{~min}$. in the chamber removed and replaced by inactive dry air. The activity of the air amounted to several microcuries.

The residual activity of the chamber after the active air has been removed gave a typical curve $(A$, Fig. 2) of the radon-active deposit of rapid change, with no trace of the thoron-active deposit.

This experiment has been repeated in a different manner. When filling the ionization chamber the air current was passed directly upon the tube with radioactive salts enclosed in a glass tube of slightly larger diameter. With other conditions identical with those of the previous experiments, the residual activity of the ionization chamber (curve $B$, Fig. 2) gave after four hours the well-known plateau of the active deposit of thoron.

In order to find if the thoron is able under normal conditions to escape from the tube at all, a tin-foil (F, Fig. 1) was wrapped directly on the tube and left there for $5 \mathrm{~min}$. The activity of the tube was then measured by means of alpha rays. A decay curve 